\title{
Broadband converted phases from midmantle discontinuities
}

\author{
Lev Vinnik ${ }^{1,2}$, Fenglin Niu ${ }^{1}$, and Hitoshi Kawakatsu ${ }^{1 *}$ \\ ${ }^{1}$ Earthquake Research Institute, University of Tokyo, 1-1-1 Yayoi, Bunkyo-ku, Tokyo 113-0032, Japan \\ ${ }^{2}$ Institute of Physics of the Earth, Moscow, Russia
}

(Received May 7, 1998; Revised September 10, 1998; Accepted September 23, 1998)

\begin{abstract}
A technique for detecting intermediate-period (6-12 s) $S d P$ phases converted from $S$ to $P$ at a depth $d$ in the source region is described. Previously, these phases were detected in short-period array recordings of deep events. The main idea of our technique is to deconvolve the vertical component of a single record by the $S$ waveform, and to stack the deconvolved components of a number of records, with appropriate time-shift corrections accounting for the difference of epicentral distance. Using this technique, the phases converted from discontinuities at around $660 \mathrm{~km}$, $860 \mathrm{~km}, 1070 \mathrm{~km}$, and $1170 \mathrm{~km}$ depths beneath Sunda arc are detected at seismograph stations in central and eastern Asia. Our data on ' $1070 \mathrm{~km}$ ' discontinuity are very consistent with those inferred from short-period recordings of the same events at the J-array in Japan (Niu and Kawakatsu, 1997), but favour a few different discontinuities in the midmantle, rather than one with a strongly variable depth. When compared with a tomographic model of the mantle for the same region, our data suggest that ' $1070 \mathrm{~km}$ ' discontinuity may act as a barrier for the downgoing lithospheric slabs.
\end{abstract}

\section{Introduction}

Observations of seismic phases converted in the mantle from $P$ to $S$ and from $S$ to $P$ provide valuable data on the properties of the mantle discontinuities. The $S$ waves can be converted to $P$ either in the source or in the receiver region. We consider the $S d P$ phases converted from $S$ to $P$ at a depth $d$ in the source region and recorded in the tail of $P$ (Fig. 1). To distinguish them from the waves reflected above the source, the latter are termed $s D P$, where $D$ is the depth of the reflector. Mantle $S d P$ phases can be detected in short-period (1-2 s) array recordings of deep events (Barley et al., 1982). These observations were used to infer topography on $660 \mathrm{~km}$ discontinuity in subduction zones (e.g., Bock and Ha, 1984; Richards and Wicks, 1990; Vidale and Benz, 1992; Wicks and Richards, 1993; Niu and Kawakatsu, 1995). Estabrook et al. (1994) presented broad-band records of a deep event, where $S^{\prime} 660^{\prime} P$ could be seen practically without any processing. The $S d P$ phases related to midmantle discontinuities (850-1200 km depth range) have been identified in shortperiod array recordings (Bock and Ha, 1984; Kawakatsu and Niu, 1994; Niu and Kawakatsu, 1997). Now, we describe the technique and some results of a detection of these phases at the periods around 6-12 s.

There are at least three reasons for investigating longerperiod data. First, the short-period converted phases can not arise at discontinuities more than $10 \mathrm{~km}$ wide. Second, at longer periods, wave scattering effects are relatively weak, and small-scale scatterers can hardly be mistaken for the real discontinuities. And third, as will be shown, observations of

*To whom correspondence should be addressed.

Copy right(c) The Society of Geomagnetism and Earth, Planetary and Space Sciences (SGEPSS); The Seismological Society of Japan; The Volcanological Society of Japan; The Geodetic Society of Japan; The Japanese Society for Planetary Sciences. the intermediate-period $S d P$ phases do not require special receiver arrays. In this paper we describe our technique, with applications to the records of deep events in Indonesia (Table 1 and Fig. 2), obtained at digital seismograph stations in east Asia. Niu and Kawakatsu (1997), further on NK, processed short-period recordings of events 2-4 at the Jarray in Japan, and detected $S_{1080^{\prime}} P$. These events were relocated by NK, and in Table 1 we adopt their estimates of event depths. Locations of the seismograph stations used in our study are shown in Fig. 2.

\section{Detection Technique}

We are interested mainly in the $S d P$ phases related to discontinuities in the $850-1200 \mathrm{~km}$ depth range. In the records of deep events, these phases are polarized as $P$ and arrive with a lapse time of a few tens of seconds relative to $P$. A delay of $10 \mathrm{~s}$ is roughly equivalent to $100 \mathrm{~km}$ in depth. The optimum width of the time window for observing these phases is in the epicentral distance range between $30^{\circ}$ and $50^{\circ}$. At smaller distances, the $S d p$ phases with $d$ more than $1000 \mathrm{~km}$ do not exist, and, moreover, the wavefield is contaminated by the late $P$ arrivals corresponding to the major upper mantle discontinuities. At distances exceeding $50^{\circ}$, the time interval between $P$ and $P c P$ becomes prohibitively small. The optimum source depths are $450-660 \mathrm{~km}$.

Our technique is akin to that developed for detecting mantle $P s$ phases (Vinnik, 1977). The record is decomposed in the vertical $(Z), \operatorname{radial}(R)$ and transverse ( $T$ or $S H$ ) components, low-pass filtered and deconvolved by the $S$ waveform in the same record. The $S$ waveform is transformed by deconvolution into a "bump". It is assumed that the waveform of the recorded $S V$ is similar to that, which generates $S d P$, in spite of a $25^{\circ}$ difference between their take-off angles and the effect of anelastic attenuation. In particular, due to the difference in anelastic attenuation between $S$ and $P$, the dominant 


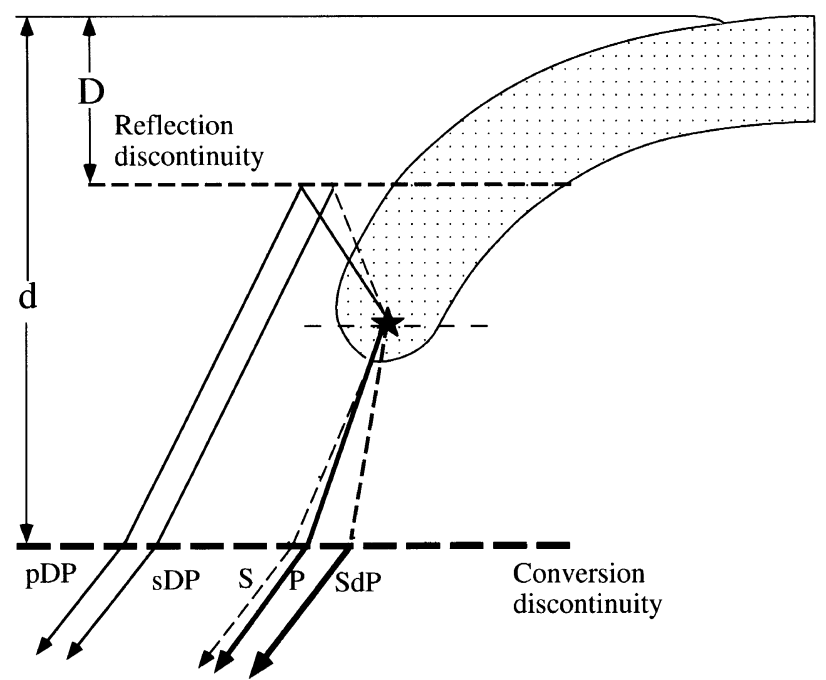

Fig. 1. Ray paths of $S d P$ and $s D P$.

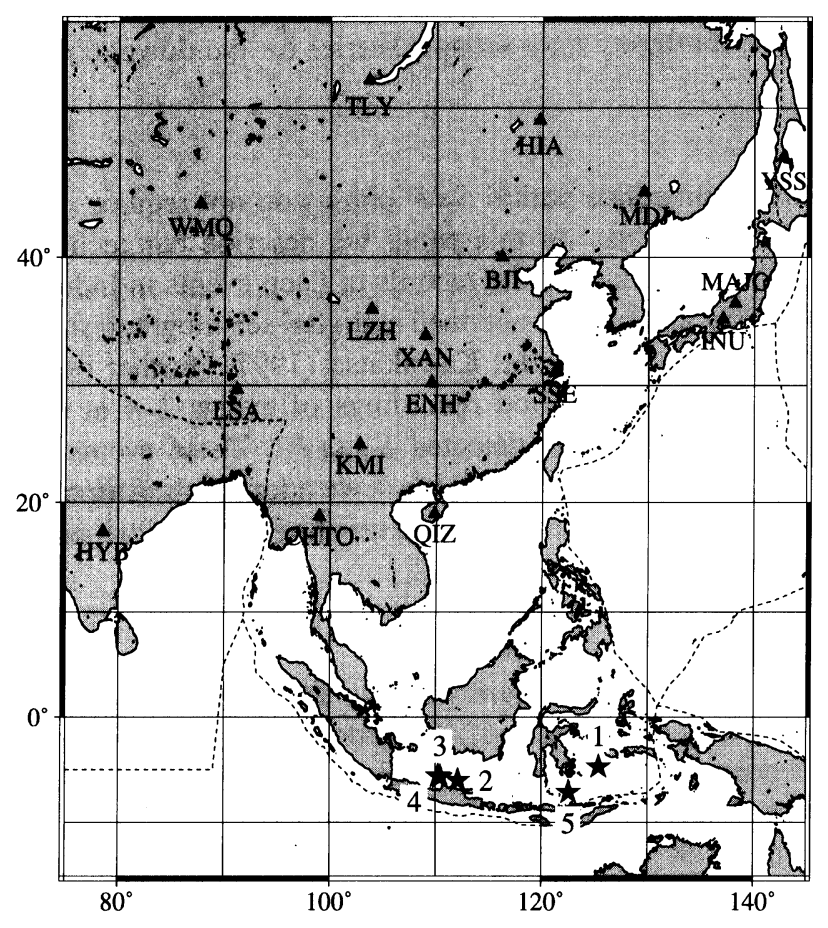

Fig. 2. Study region, with the seismograph stations and the epicenters of the seismic events.

period of $S$ (around $10 \mathrm{~s}$ ) can be longer than that of $S d P$ by a fraction of a second. For practical purposes, this difference can be neglected. A similarity between the waveforms of the recorded $S V$ and the parent $S V$ for $S d P$ is likely, if, as in our data, both are on the same side of the nodal line for $S V$ and far from it. Then the $S d P$ waveform in the $Z$ component of the record is transformed by deconvolution into a "bump", as well. This "bump" can be detected by stacking the deconvolved $Z$ components of several events at several stations, with appropriate moveout corrections. Sometimes, $S d P$ arrives in the time window, where the record of the vertical

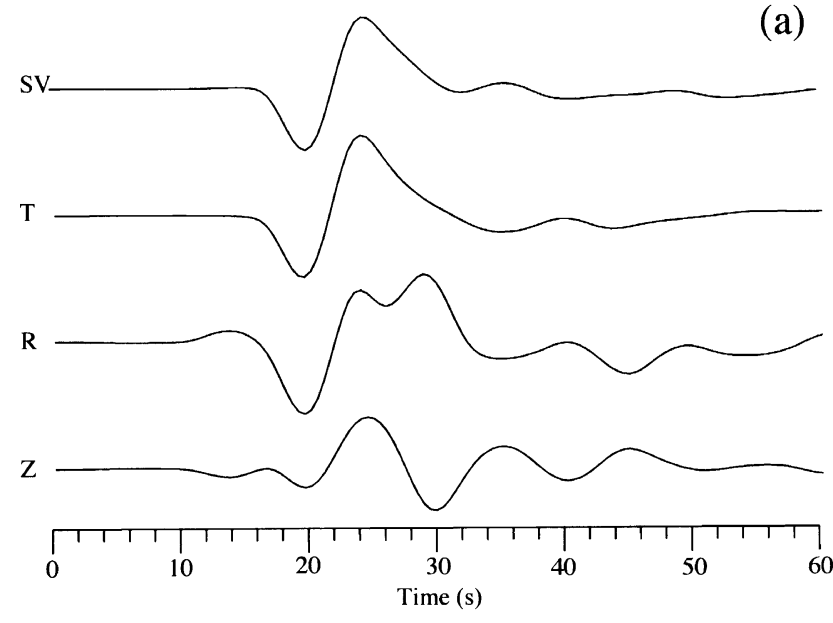

(b)
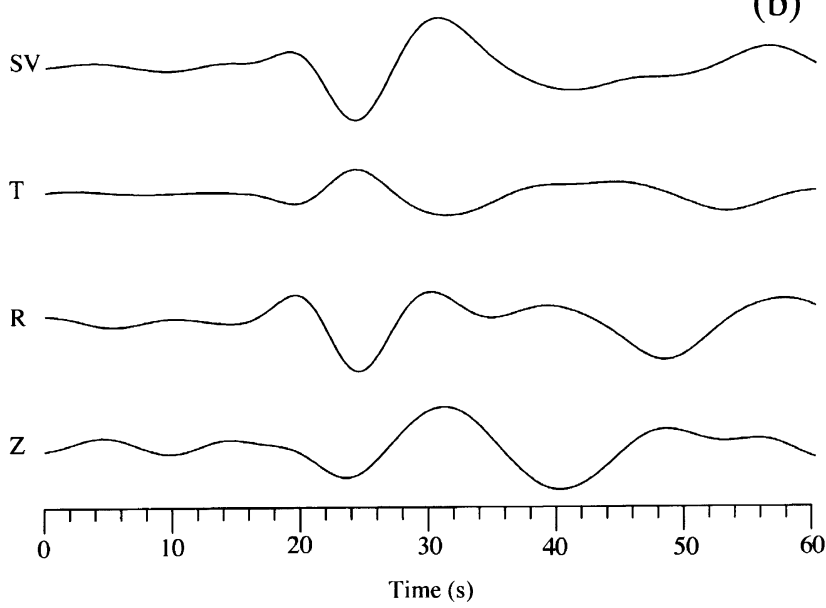

Fig. 3. Examples of synthetic (a) and real (b) records of $S$. The $S V$ component corresponds to the direction forming an angle of $40^{\circ}$ with the vertical direction in the wave propagation plane. The synthetic seismogram is calculated at an epicentral distance of $40^{\circ}$ for a model similar to IASP91, but with a $40 \mathrm{~km}$ thick crust. The real seismogram is for event 1 , station $\mathrm{LZH}, 45.3^{\circ}$ distance. Note similarity between the waveforms of $S V$ and $S H$ in both synthetic and real record.

component can be disturbed by reverberation in the crust in a vicinity of the receiver. The reverberation is polarized as $S V$. To minimize the disturbance, the records are projected on the axis $P$, parallel to the principal motion direction in the recorded $P$ wave. An angle between this axis and the vertical direction is between $20^{\circ}$ and $30^{\circ}$. In the rest of this section, we describe the processing procedure in detail.

Waveforms of $S V$ and $S H$, for the point dislocation source, are similar, except the sign and amplitude. Then, although $S d P$ is coupled with $S V$, the record can be deconvolved by either $S V$ or $S H$. If the particular horizontal component of the recorded $S$ is weak relative to the other component, its waveform can be disturbed by coupling with the other horizontal component, due to shear wave splitting in the upper mantle (Farra et al., 1991). The disturbance looks like the derivative of the stronger component. To avoid this, the stronger component, either $S V$ or $S H$, is preferable for the deconvolution.

In its turn, the $S V$ component can be distorted by coupling with $P$ in the receiver region. In the distance range of interest, 
Table 1. Parameters of the events; $\phi, \delta$, and $\lambda$ are the parameters of the fault plane solution.

\begin{tabular}{ccccccccc}
\hline Event & Date & Time & Lat. & Lon. & Depth & $\phi$ & $\delta$ & $\lambda$ \\
\hline 1 & $07 / 14 / 92$ & $07: 03: 10.7$ & -4.71 & 125.43 & 477.0 & 356 & 62 & -147 \\
2 & $09 / 02 / 92$ & $05: 50: 02.5$ & -6.04 & 112.14 & 636.5 & 291 & 34 & -79 \\
3 & $09 / 28 / 94$ & $16: 39: 52.0$ & -5.67 & 110.46 & 638.0 & 319 & 50 & -69 \\
4 & $11 / 15 / 94$ & $20: 18: 11.3$ & -5.60 & 110.23 & 570.0 & 311 & 47 & -62 \\
5 & $06 / 17 / 96$ & $11: 22: 18.5$ & -7.14 & 122.59 & 587.0 & 225 & 50 & -131 \\
\hline
\end{tabular}

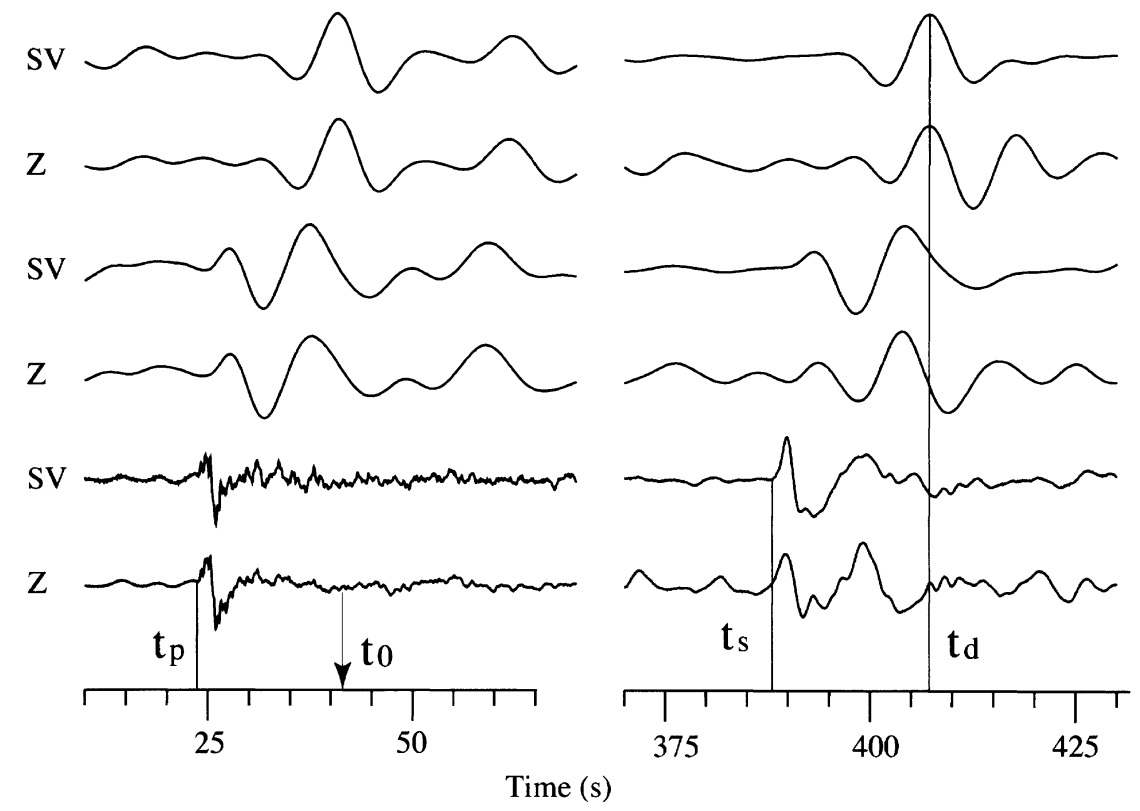

Fig. 4. Illustration of data processing for event 1, station LZH: two initial traces (bottom), the same traces but low pass filtered (middle), and the same traces low pass filtered and deconvolved by $S V$ (top). All traces are plotted with equal amplitudes.

the apparent velocity of $S$ is close to $7.5 \mathrm{~km} / \mathrm{s}$, lower than the $P$ velocity in the uppermost mantle. Hence, the $P$ wave generated at the Moho by conversion is an inhomogeneous wave, the transmission coefficient for $S V$ is complex, and the waveform of the transmitted $S V$ is distorted relative to the incoming wave (Aki and Richards, 1980). We investigated this effect in synthetic seismograms, calculated with the aid of reflectivity technique (Fuchs and Mueller, 1971; Kind and Mueller, 1975). Figure 3(a) demonstrates the synthetic at a distance of $40^{\circ}$ for the model, similar to IASP91 (Kennett and Engdahl, 1991), but with a thicker crust (40 km), appropriate for the continent. The waveform in the radial component $R$ is strongly different from the transverse component $T$, but, if the seismogram is projected on the axis forming an angle of $40^{\circ}$ with the vertical direction in the wave propagation plane, the difference becomes insignificant. The angle of $40^{\circ}$ is found empirically. The resulting component is practically free from the $P$ wave motion and close to $S V$. Further on, it is termed $S V$. Figure 3(b) demonstrates a similar effect in the real data.

Let the arrival times of $P$ and $S$ be $t_{p}$ and $t_{s}$, and the maximum displacement in the deconvolved $S$ be at $t_{d}$ (Fig. 4). We define the delay time of $S d P$ relative to $P$ via the time of the maximum displacement in the deconvolved $S d P$. Then $t_{0}$, origin of the time scale for the delay of $S d P$, is defined as the time of the maximum displacement in $S d P$ for the conversion depth coinciding with the source depth: $t_{0}=t_{p}+\left(t_{d}-t_{s}\right)$. To determine $t_{0}$ accurately, $t_{p}$ and $t_{s}$ should be measured with the accuracy of a fraction of a second, but in some of the low-pass filtered records, this can be difficult. However, the expression for $t_{0}$ can be written as $t_{0}=\left(t_{p}-t_{s}\right)+t_{d}$, and the differential time $\left(t_{p}-t_{s}\right)$ can be determined with the maximum accuracy at shorter periods. Our experiments with the best records demonstrate that $\left(t_{s}-t_{p}\right)$ at the periods around $10 \mathrm{~s}$ is by about $1 \mathrm{~s}$ larger than in the short-period range, due mainly to the physical dispersion of $S$ in the upper mantle. However, for $S d P$, the length of the wave-path of $S$ in the upper mantle is much shorter, and this effect, already small, should be even smaller.

The expected amplitudes of the $S d P$ phases from midmantle discontinuities can be in the range of $1 \%$ of the amplitude of $S V$. To detect them reliably, the events with a specific radiation pattern are preferable. Figure 5 shows theoretical amplitudes of $P$ and $S V$ radiated from event 4 of 


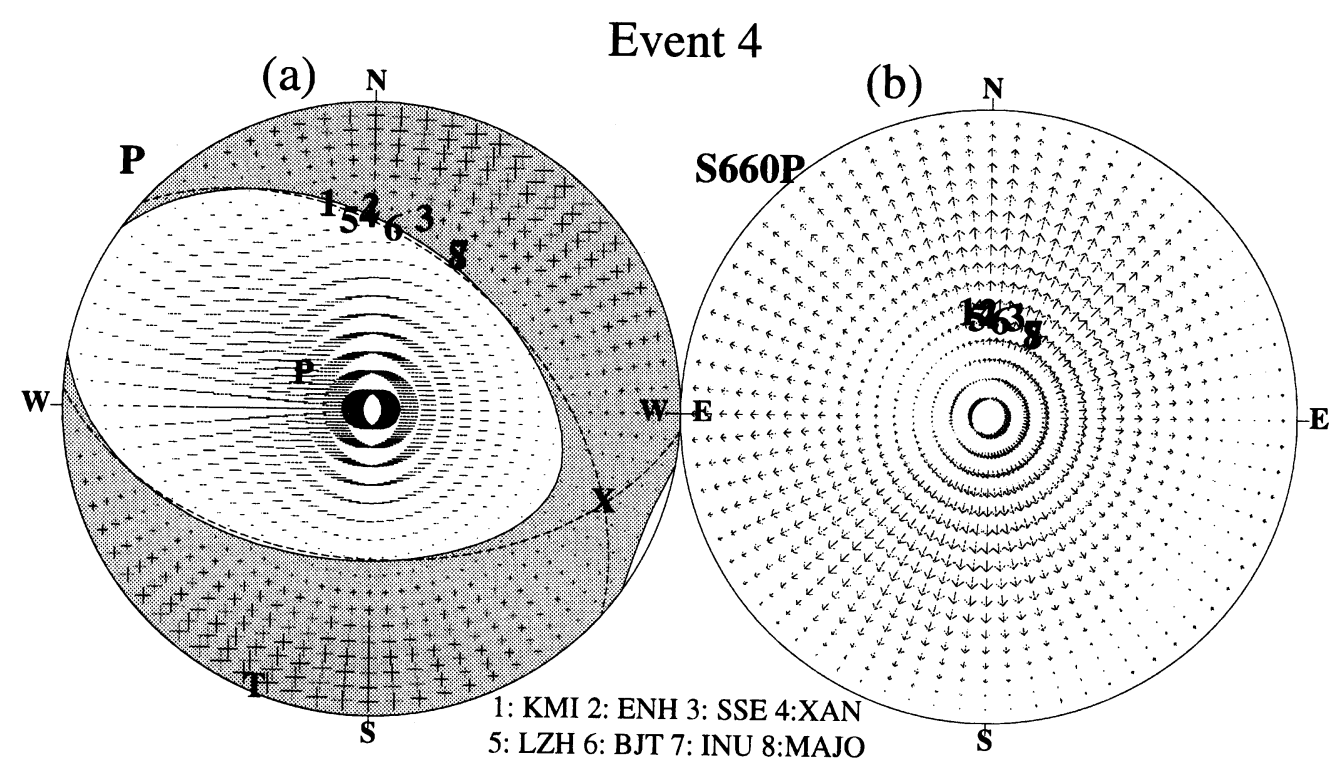

Fig. 5. Theoretical amplitudes of $P$ (a) and $S V$ (b) radiated from event 4 . Each point on the Earth's surface is specified by its azimuth relative to the epicenter and the incidence angle in the source for either $P$ (a) or $S d P(b)$. Note, that our seismograph stations are located in a vicinity of the nodal line for $P$, where the expected amplitudes of $P$ are small, whereas those of $S d P$ are close to the largest possible.

Table 1. Our seismograph stations are located in a vicinity of the nodal line for $P$, where the expected amplitudes of $P$ are small, whereas those of $S d P$ are close to the largest possible. Similar radiation patterns are characteristic of the other events in Table 1.

\section{Testing the Technique: Detection of $S_{660} P$}

According to the literature, ' $660 \mathrm{~km}$ ' discontinuity is present in every region, where the analysis of seismic data data was performed. Detecting $S^{\prime} 660^{\prime} P$ is simplified by the large $S$ velocity contrast at ' $660 \mathrm{~km}$ ' discontinuity, and, consequently, by the large amplitude of $S d P$. On the other hand, a relatively small time lag of $S d P$ relative to $P$ complicates the problem. The optimum conditions for detecting $S^{\prime} 660^{\prime} P$ (suitable source depth and radiation pattern) are provided by event 1 of Table 1 . The raw records are integrated to obtain displacement, low-pass filtered with a corner period at $10 \mathrm{~s}$, and deconvolved, as described in preceding section. Deconvolution is performed in time domain, with a proper regularization. The amplitudes are normalized to the amplitude of the recorded $S V$, corrected for the theoretical source radiation pattern. Moveout corrections for stacking are calculated by multiplying the assumed differential slowness by the differential distance. The differential slowness is determined relative to $P$, and the differential distance is determined relative to the reference distance. The reference distance corresponds roughly to the average epicentral distance, and in our calculations is fixed at $42^{\circ}$.

Figure 6(a) demonstrates the $P$ components at a number of stations, prior to stacking. The second strong arrival (marked by arrows) at a time around $19 \mathrm{~s}$ is, most likely, $S_{660^{\prime}} P$. The times of this phase at most stations fluctuate with an amplitude not more than about $1 \mathrm{~s}$. Station HYB is exceptional: $S^{\prime} 660^{\prime} P$ at HYB arrives $5 \mathrm{~s}$ later than at the other stations. This phenomenon is discussed in the last section.
Figure 6(b) shows the stack of all traces shown in Fig. 6(a), except HYB. The assumed $S^{\prime} 60^{\prime} P$ is very clear in the stack, with a time of $19.2 \mathrm{~s}$ and the largest amplitude $(6 \%$ of $S V)$ at a slowness around $-0.1 \mathrm{~s} /{ }^{\circ}$.

Numerical simulation of the data in Fig. 6(b) is shown in Fig. 6(c). The synthetics were calculated with reflectivity technique for a point source with the focal parameters of event 1 , for $330^{\circ}$ azimuth in $34^{\circ}-50^{\circ}$ distance range, and processed like the real seismograms. The adopted azimuth and distance range are representative of the available data for event 1. Two models used are IASP91 (Kennett and Engdahl, 1991), and a modified IASP91, without a sharp transition at $410 \mathrm{~km}$ depth. The latter model allows to assess the contribution from $S^{\prime} 410^{\prime} P$, which arrives at about the same time as $S^{\prime 660^{\prime}} P$. Both models are without anelastic attenuation. For $-0.1 \mathrm{~s} /{ }^{\circ}$ slowness, the record of $S^{\prime} 660^{\prime} P$ is practically similar to $S_{660^{\prime}} P+S^{\prime} 410^{\prime} P$, which implies that $S^{\prime} 410^{\prime} P$ is much weaker than $S_{660^{\prime}} P$. Other data (see next section) suggest that in the actual data $S^{\prime} 410^{\prime} P$ is even weaker than in the synthetics. The amplitude of $S_{660^{\prime}} P$ in the synthetics is close to that in the actual data, whereas its time $(18.0 \mathrm{~s})$ is smaller by $1.2 \mathrm{~s}$. Part of the difference can be attributed to a thin $(35 \mathrm{~km})$ crust in IASP91. The rest may correspond to a difference of several kilometres between the depths of the discontinuity in IASP91 and in our data, or to an error of several kilometres in the event depth. Figure 6(c) demonstrates that, relative to $S d P, P$ in the synthetic stack is much stronger than in the actual data. This might be a result of a stronger correlation between the waveforms of $P$ and $S$ in the synthetics than in the actual data.

\section{Observations of $S d P$ from Midmantle Disconti- nuities}

Successful detection of $S_{660^{\prime}} P$ justifies applications of the same technique for detecting weaker signals from midmantle 


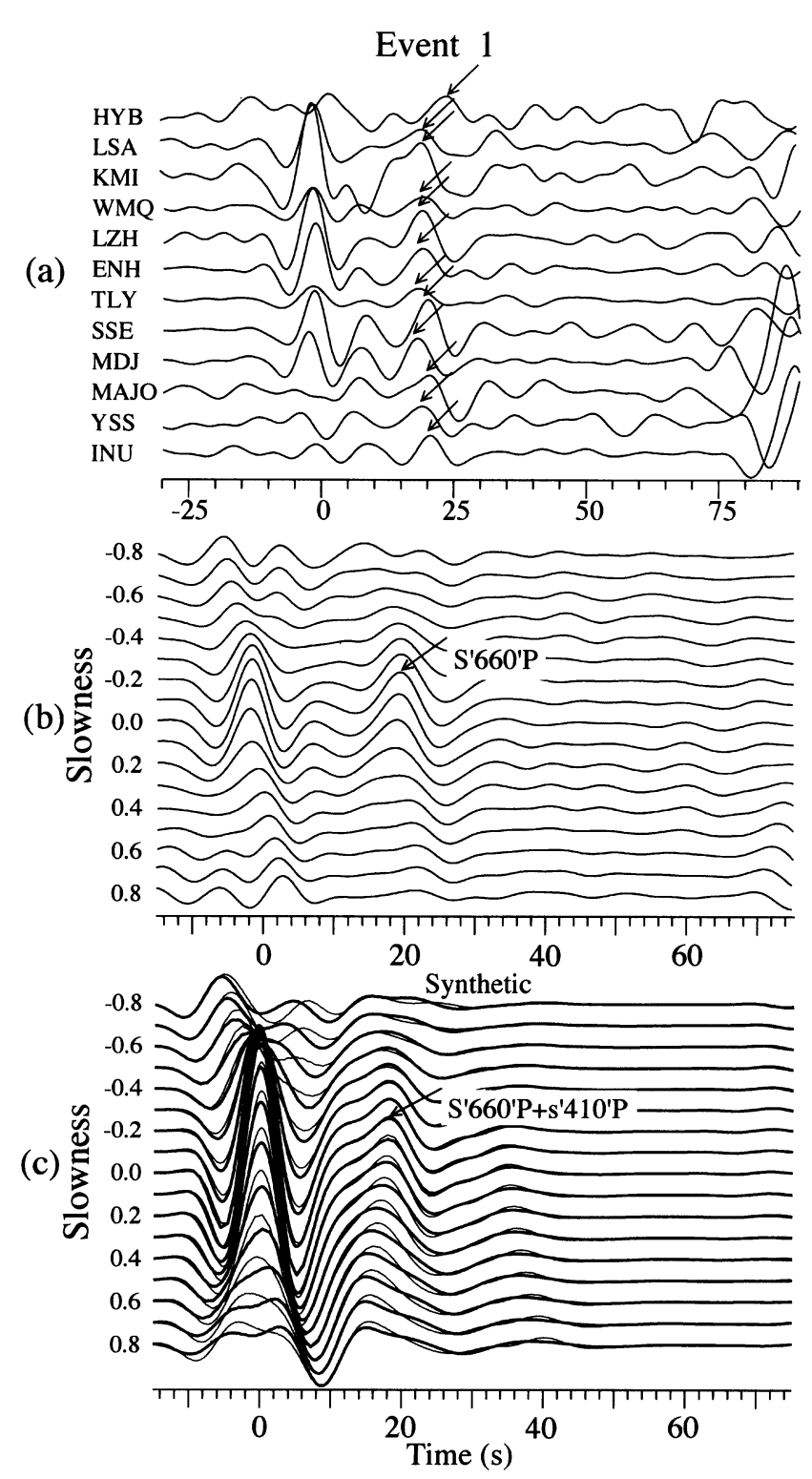

Fig. 6. Detection of $S^{\prime} 660^{\prime} P$ in the records of event 1. (a) Deconvolved $P$ component traces; the stations are arranged according to the azimuth from the epicenter, from $-63.3^{\circ}$ at HYB to $14.4^{\circ}$ at INU; origin of the time scale corresponds to $t_{0}$. (b) Stacks of all traces shown in (a), except HYB; differential slowness in $\mathrm{s} /{ }^{\circ}$ is shown on the left. (c) The same as (b), but for the synthetic seismograms calculated for the focal mechanism and depth of event 1. The traces for IASP91 earth model (Kennett and Engdahl, 1991) are shown by bold lines. The traces shown by thin lines are obtained for the same model, but without a sharp boundary at $410 \mathrm{~km}$. The phase marked by an arrow is either $S^{\prime} 660^{\prime} P+S^{\prime} 410^{\prime} P$ for IASP91, or $S^{\prime} 660^{\prime} P$ for the modified IASP91.

discontinuities. There are a few criteria to distinguish weaker but true signals from artifacts of stacking. Sometimes an arrival in the slowness stack is caused by anomalously large amplitude at one or two neighbouring stations, rather than by constructive interference of signals in the records of all stations. Characteristic features of such arrivals in the stack are weak dependence of amplitudes and, often, strong dependence of their times on slowness. By comparison, the true signals can be distinguished by (1) a strong dependence of the amplitude on slowness and (2) by a stable time in the slow- (a)

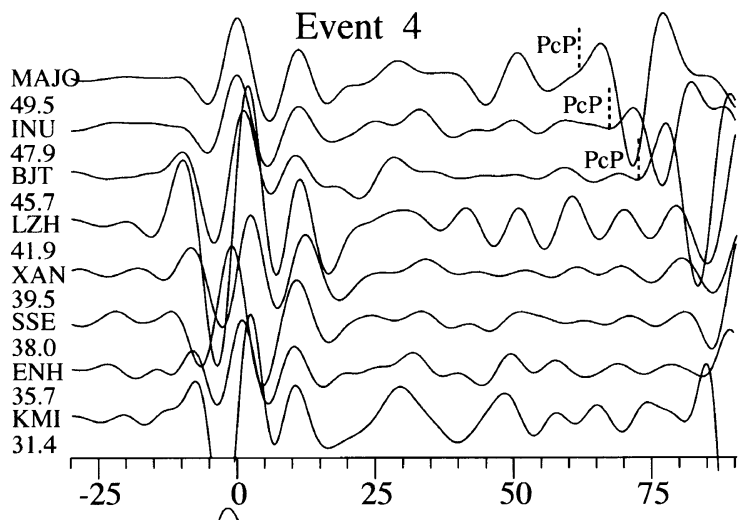

(b)

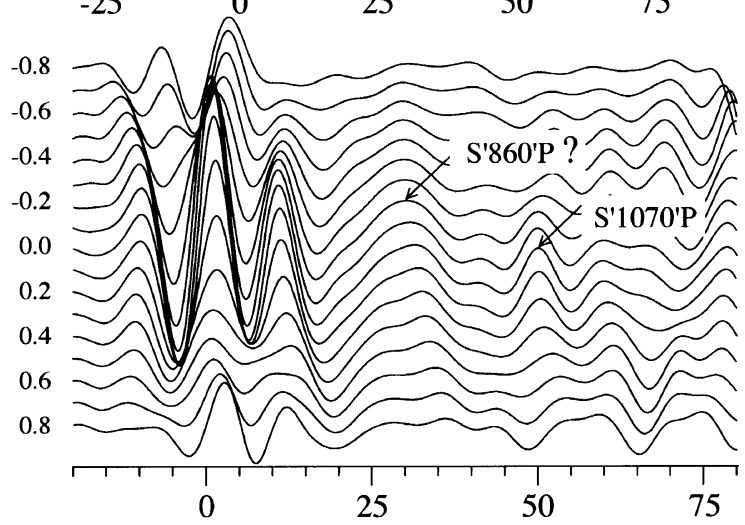

(c)

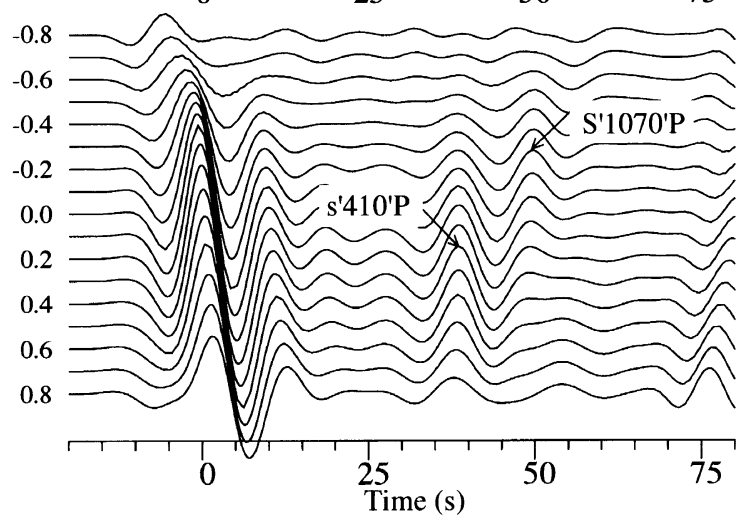

Fig. 7. Data for event 4. (a) Deconvolved $Z$ component traces; epicentral distances in degrees are shown on the left; origin of the time scale corresponds to $t_{0}$. (b) Stacks of traces in (a); differential slowness in $\mathrm{s} /{ }^{\circ}$ is shown on the left. (c) The same as in (b), but for the reflectivity synthetics calculated for the focal mechanism and depth of event 4 .

ness range corresponding to the largest amplitudes. In our stacking procedure, the sum of individual traces is normalized by dividing it by the number of traces. To be detected reliably, the signal in the stack should be (3) comparable in amplitude with the individual traces in the corresponding time window. Finally, (4) the signal in the stack should be preceded by a quiet interval at least $10 \mathrm{~s}$ long, where the amplitude is a few times lower. The signals described in the rest of this section satisfy either all criteria, or, at least, the first three of them. Moreover, we will demonstrate that the signals thus detected are present consistently in various data sets.

The observations are modelled with reflectivity techniques. The actual fault plane solution and depth, as well as representative azimuth and distance range are adopted for 

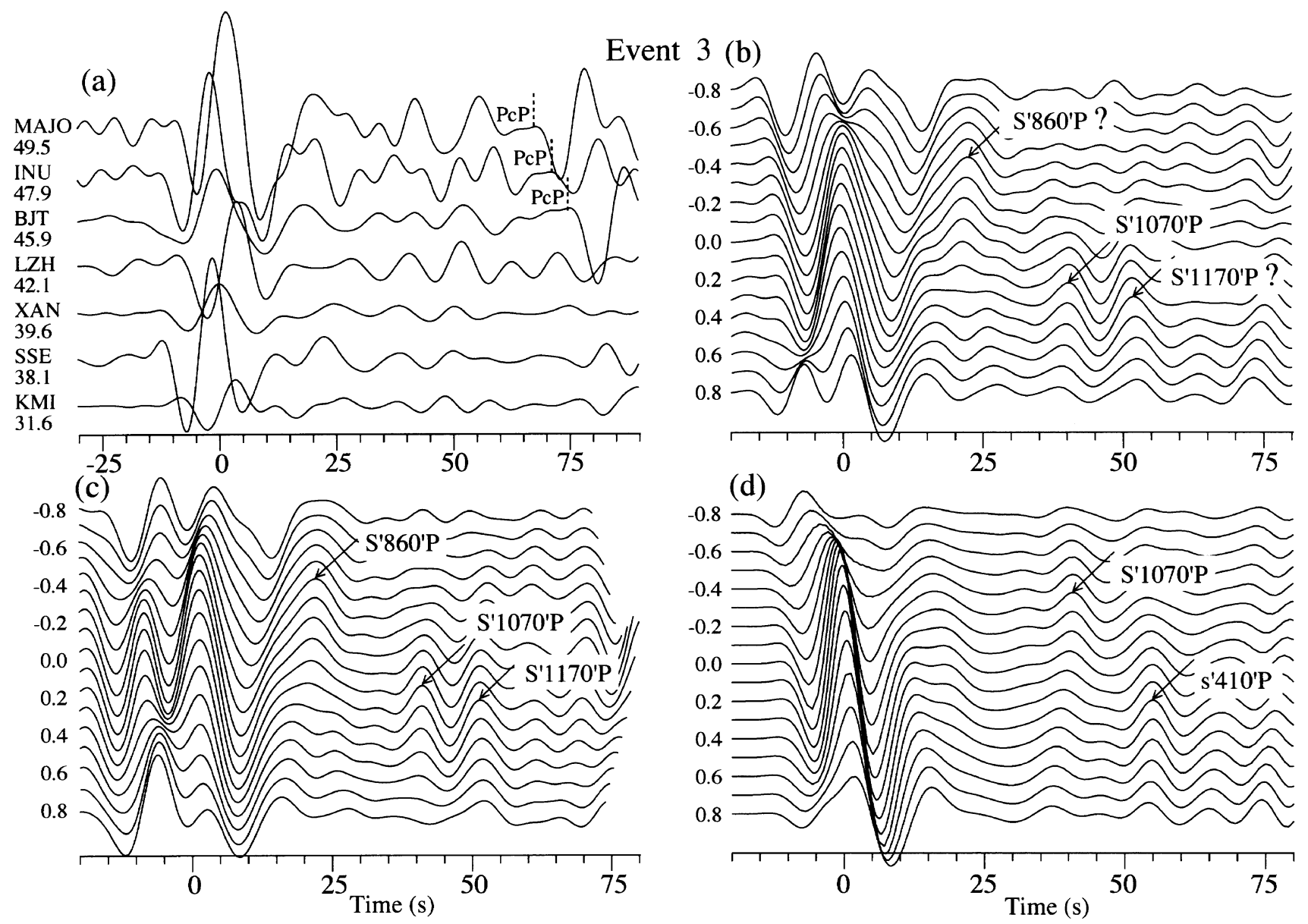

Fig. 8. Data for event 3. (a) Deconvolved $P$ component traces; epicentral distances in degrees are shown on the left; origin of the time scale corresponds to $t_{0}$. (b) Stacks of traces in (a); differential slowness in $\mathrm{s} /{ }^{\circ}$ is shown on the left; (c) The same as in (b), but for the traces in (a) and those in Fig. 7(a); the latter are shifted by $-9 \mathrm{~s}$, to account for the difference in the depths of the events. (d) The same as in (b), but for the reflectivity synthetics calculated for the focal mechanism and depth of event 3.

each event. The synthetics are calculated for IASP91 with an additional discontinuity: $S$ velocity rising with depth by $0.2 \mathrm{~km} / \mathrm{s}$ at $1070 \mathrm{~km}$. $S_{1070^{\prime}} P$ is insensitive to other parameters of this discontinuity. No anelastic attenuation is assumed. The synthetics are processed, like the real seismograms.

We start from the analysis of data for events 2-4, previously used by NK.

Event 4: The time interval for detecting $S d P$, bounded by $P$ and $P c P$, is between about $20 \mathrm{~s}$ and $60 \mathrm{~s}$ (Fig. 7(a)). The stack (Fig. 7(b)) reveals a clear "bump" with 0.014 amplitude, $50.1 \mathrm{~s}$ time and a slowness of $0.1 \mathrm{~s} /{ }^{\circ}$. A clear signal interpreted as $S_{1080^{\prime}} P$, with a similar time is observed by $\mathrm{NK}$ in the records of this event at the J-array. In the synthetic stack (Fig. 7(c)), two secondary phases are visible. One is with a time of $49.5 \mathrm{~s}$ and an amplitude of 0.01 is $S_{1070^{\prime}} P$, and the other, with an amplitude of 0.02 at a time of $38.3 \mathrm{~s}$ is $S^{\prime} 410^{\prime} P$. The largest amplitudes of $S_{1070^{\prime}} P$ and $S^{\prime} 410^{\prime} P$ are obtained for the differential slowness values of $-0.1 \mathrm{~s} /{ }^{\circ} \sim-0.2 \mathrm{~s} /{ }^{\circ}$ and $0.3 \mathrm{~s} /{ }^{\circ}$, respectively. The phase with a time around $50 \mathrm{~s}$ is present in Fig. 7(b), though with a different slowness, whereas the second one, in spite of its strength in the synthetics, is missing. $10 \mathrm{~km}$ in depth of the event is equivalent to $2.7 \mathrm{~s}$ in time of $s D P$. To identify the phase with $50.1 \mathrm{~s}$ time in Fig. 7(b) with $S^{\prime} 410^{\prime} P$, the actual depth of the event should differ from that in Table 1 by a few tens of kilometres, which is unlikely. Then, this phase should be interpreted as $S_{1070^{\prime}} P$. A discrepancy between the observed and theoretical slowness of this phase is documented for the other events, as well, and is discussed in last section of the paper. Another "bump" in Fig. 7(b), with a slowness around $0.0 \mathrm{~s} /{ }^{\circ}$, and a time around $30 \mathrm{~s}$ is remarkable by its long period. This can be $S^{\prime} 860^{\prime} P$.

Event 3: Event 3 is located very closely to event 4 , but the depth is different ( $638 \mathrm{~km}$ versus $570 \mathrm{~km})$. The interval for detecting the converted phases in the individual $P$ component traces is limited by $20 \mathrm{~s}$ and $65 \mathrm{~s} \mathrm{(Fig.} \mathrm{8(a)).} \mathrm{In} \mathrm{the}$ resulting stack (Fig. 8(b)), two "bumps" are clearly visible: one with 0.013 maximum displacement at $0.2 \mathrm{~s} /{ }^{\circ} \sim 0.3 \mathrm{~s} /{ }^{\circ}$ slowness and $40.4 \mathrm{~s}$ time, and the other, with 0.017 maximum displacement at $0.4 \mathrm{~s} /{ }^{\circ}$ slowness and $51.8 \mathrm{~s}$ time. A third "bump" with 0.022 maximum displacement at $-0.3 \mathrm{~s} /{ }^{\circ}$ slowness and $21.4 \mathrm{~s}$ time is less obvious, due to the neighbourhood of $P$. If the signals in Fig. 8(b) are the $S d P$ phases, they should arrive with a delay of 8-9 s relative to those in Fig. 7. To check this, the records in Fig. 7(a) are shifted by 
$-9 \mathrm{~s}$ and stacked with those in Fig. 8(a). The three phases which are visible in Fig. 8(b) are enhanced in the composite stack (Fig. 8(c)). Explanation of $40.4 \mathrm{~s}$ signal in Fig. 8(b) as $S_{1070^{\prime}} P$ is supported by observation of $S_{1080^{\prime}} P$ with a similar time in the records of this event at the J-array by NK. The two other "bumps" can be interpreted as $S_{860^{\prime}} P$ and $S_{1170^{\prime}} P$. In the synthetic stack (Fig. 8(d)), the maximum displacement in $S_{1070^{\prime}} P(0.011)$ is observed at $41.3 \mathrm{~s}$ time and $-0.3 \mathrm{~s} /{ }^{\circ}$ slowness. The other "bump" with the maximum displacement $(0.014)$ at $53.5 \mathrm{~s}$ time and $0.3 \mathrm{~s} /{ }^{\circ}$ slowness is $S^{\prime} 410^{\prime} P$. This phase, dominant in the synthetics, is either missing in the real records, like in the records of event 4 , or interferes constructively with $S_{1170^{\prime}} P$.

Event 2: Epicenter of event 2 is located $2^{\circ}$ eastward of events 3 and 4 . Its depth $(636.5 \mathrm{~km})$ is very close to that of event 3 , their focal mechanisms are practically similar, and the synthetics for event 3 are applicable to event 2 . The deconvolved $P$ component traces and the results of stacking are displayed in Figs. 9(a) and (b). In general, the wavefield in Fig. 9 looks similar to that in Fig. 8, but the "bumps" at $40.4 \mathrm{~s}$ and $51.8 \mathrm{~s}$ in Fig. 8 are shifted to $34.6 \mathrm{~s}$ and $45.3 \mathrm{~s}$ in Fig. 9. The largest amplitudes of the "bumps" ( 0.025 and 0.021 ) in Fig. 9 are observed at the same slowness (around $0.3 \mathrm{~s} /{ }^{\circ}$ ), as in Fig. 8. Using the same arguments, as for the records of event 3, the first "bump" can be interpreted as $S_{1070^{\prime}} P$, but with $1070 \mathrm{~km}$ discontinuity uplifted by $60 \mathrm{~km}$. $S_{1080^{\prime}} P$ with a similar time is identified in the records of this event at the J-array by NK. Identification of the second "bump" with $s^{\prime} 410^{\prime} P$ is practically impossible, due to $8.2 \mathrm{~s}$ difference in their times. More likely, this feature is similar to $S_{1170^{\prime}} P$ in the records of event 3 , with $1170 \mathrm{~km}$ discontinuity uplifted by $60 \mathrm{~km}$. The presence of a long-period "bump" corresponding to $860 \mathrm{~km}$ discontinuity, with an amplitude of 0.014 at a time of $22.7 \mathrm{~s}$ and a slowness of $-0.3 \mathrm{~s} /{ }^{\circ} \sim$ $-0.4 \mathrm{~s} /{ }^{\circ}$ can be suspected in Fig. 9 . This discontinuity in the region of event 2 seems to be at the same depth as in the neighbouring region to the west.

Event 1: This event occurred about $15^{\circ}$ to the east of events $2-4$, at a shallower depth. Records of event 1 were already inspected in Fig. 6 for the presence of $S^{\prime} 660^{\prime} P$. Now, we inspect them for signals from midmantle discontinuities. The useful time interval is terminated at $65-70 \mathrm{~s}$ by arrivals of $p P, P P$ and $P c P$ (Fig. 10(a)). In the stack (Fig. 10(b)), the phase with the maximum amplitude $(0.011)$ at $58.8 \mathrm{~s}$ time and $0.0 \mathrm{~s} /{ }^{\circ}$ slowness can be interpreted as $S_{1070^{\prime}} P$. The other signal in Fig. 10(b), with a time of 70.4 s, looks like $S_{1170^{\prime}} P$, but in this time interval, the traces of SSE, MAJO, and LSA are contaminated by $P c P, p P$, and $P P$. To check robustness of detection, these traces were dropped, but in the resulting stack (Fig. 10(c)), the same signals are seen, as in Fig. 10(b). In the synthetic stack (Fig. 10(d)), $s^{\prime} 410^{\prime} P$ arrives simultaneously with $S^{\prime} 660^{\prime} P$, and can not be mistaken for a signal from the lower mantle. The maximum amplitude (0.019) of $S_{1070^{\prime}} P$ in the synthetics is obtained at $58.8 \mathrm{~s}$ and $-0.3 \mathrm{~s} /{ }^{\circ} \sim-0.4 \mathrm{~s} /{ }^{\circ}$ slowness. The times of $S_{1070^{\prime}} P$ are very similar in the synthetics and the actual data, but the synthetic signal is almost twice stronger than the observed one. The relative weakness of the observed $S_{1070^{\prime}} P$ can also be established by comparing the amplitude ratio between $S_{660^{\prime}} P$ and $S^{\prime} 1070^{\prime} P$, in the synthetics and the actual data (4
Event 2

(a)

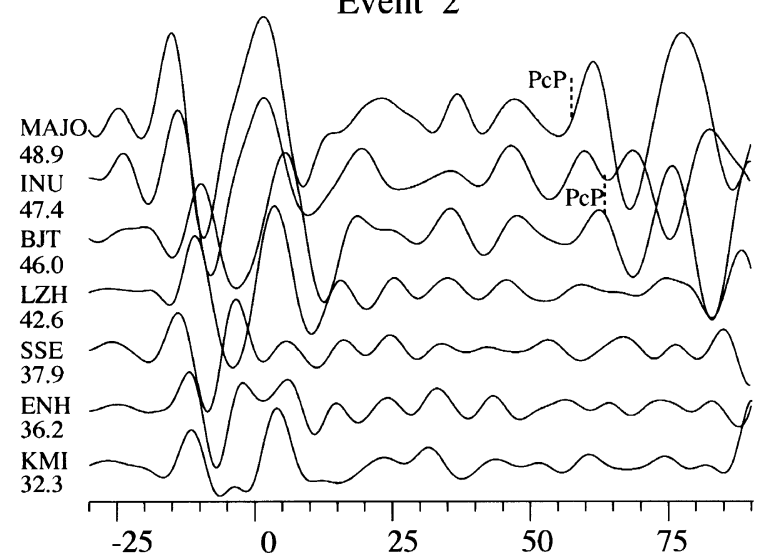

(b)

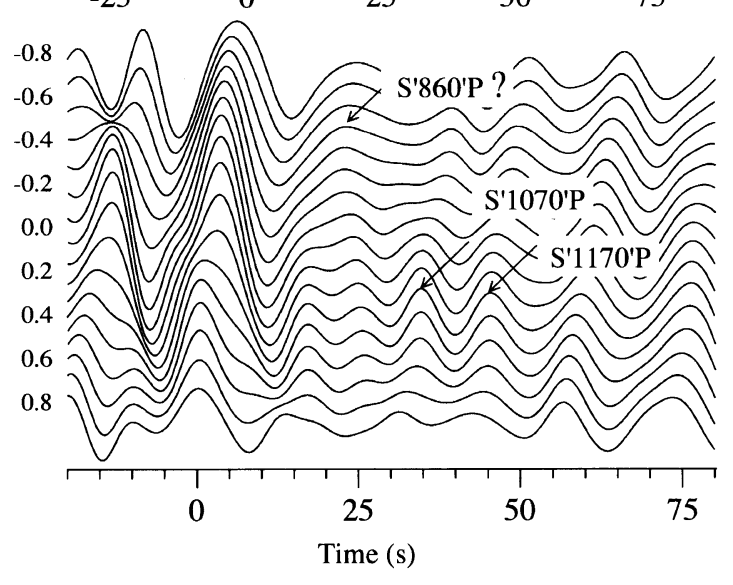

Fig. 9. The same as in Figs. 8(a) and (b), but for the records of event 2.

versus 7).

Event 5: This is the great Flores Sea deep earthquakes. Various authors have modelled its source and documented a complicated rupture history (e.g., Goes et al., 1997), which implies a time dependent $S V / S H$ relationship. To avoid this problem, the records were deconvolved only by $S V$. This event provides the minimum number of usable records (Fig. 11(a)). In the stack (Fig. 11(b)), there is a signal at $58.0 \mathrm{~s}$ with an amplitude of 0.009 . The strongest phase in the synthetics (Fig. 11(c)) is $s_{410^{\prime}} P$, with a time of $42.5 \mathrm{~s}$ and an amplitude of 0.022 , which is clearly missing in Fig. 11(b). $S_{1070^{\prime}} P$, which arrives in the synthetics at $47.3 \mathrm{~s}$, is also missing in Fig. 11(b), but the phase with a time of $58.0 \mathrm{~s}$ is a good candidate for $S_{1170^{\prime}} P$. We note that, as reported by NK, the phase similar to our $S_{1070^{\prime}} P$ is missing also at the $\mathrm{J}$-array in the records of events neighbouring to event 5 .

\section{Discussion and Conclusions}

We have described the technique for detecting $S d P$ phases with a period around $10 \mathrm{~s}$, converted in the mantle of the source region from $S$ to $P$. Efficacy of the technique was demonstrated by applying it to the records of event 1 for detecting $S_{660^{\prime}} P$. The depth of ' $660 \mathrm{~km}$ ' discontinuity thus found at most stations differs from the standard depth by several kilometres. Since this estimate is made relative to the source depth, the accuracy of which is probably in the same range, our data do not suggest any significant deviation from the normal depth of ' $660 \mathrm{~km}$ ' discontinuity in most of the 

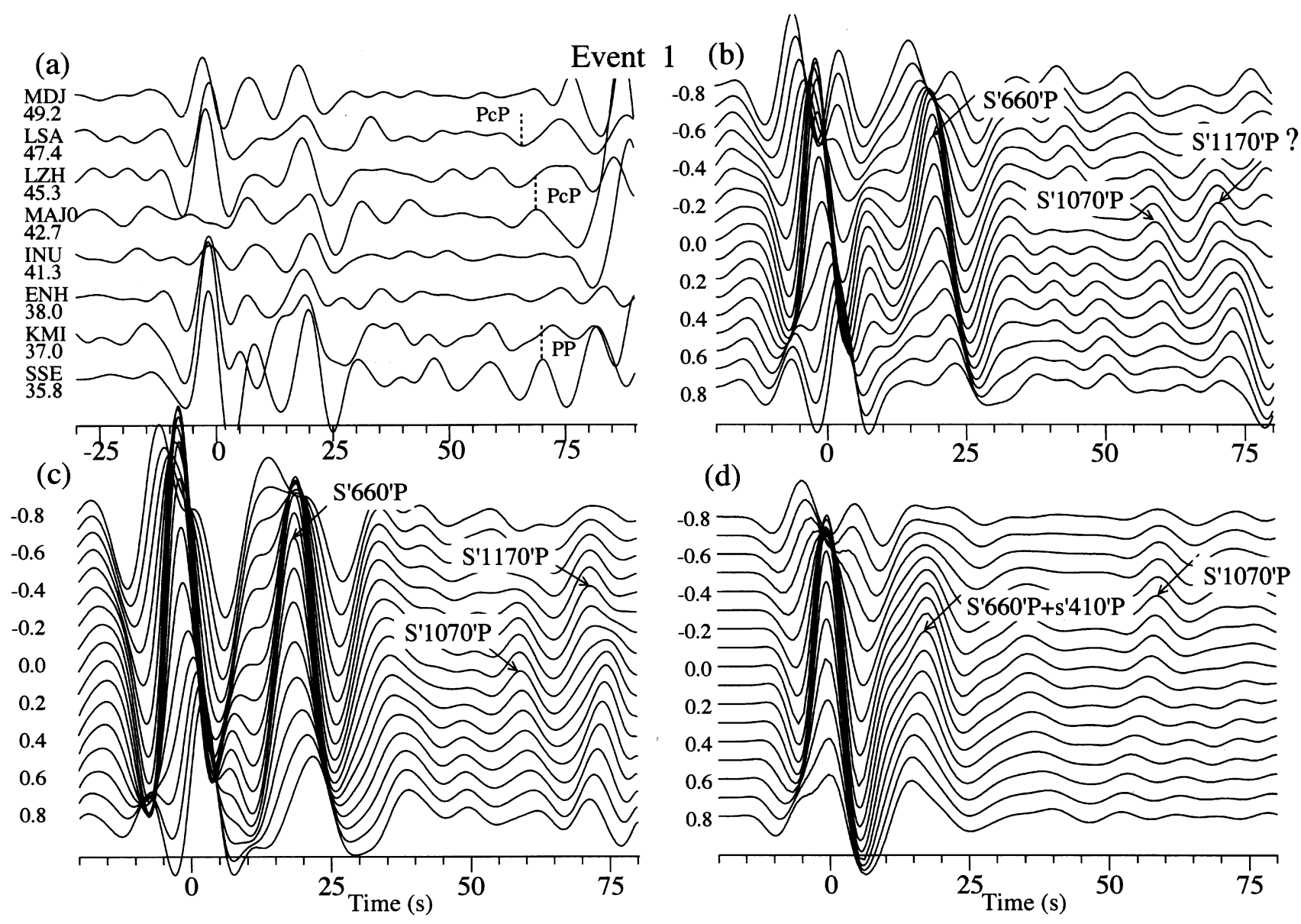

Fig. 10. Detection of the signals from midmantle discontinuities in the records of event 1. (a) The same as in Fig. 6(a), but for the stations at distances less than $50^{\circ}$, arranged according to the epicentral distance. (b) Stacks of traces in (a); differential slowness in $\mathrm{s} /{ }^{\circ}$ is shown on the left. (c) The same as in (b), but without MAJO, LSA, SSE, and with additional low-pass filtering with a corner period at 9 s. (d) The same as in (b), but for reflectivity synthetics calculated for the focal mechanism and depth of event 3.

area shown in Fig. 12 and sampled by the data. A strong anomaly (around $60 \mathrm{~km}$ relative to IASP91) is found only at station HYB. The corresponding conversion point is marked 'HYB'; the signal is formed within the first Fresnel zone with a radius of about $50 \mathrm{~km}$, around this point. The Fresnel zone for HYB may overlap a currently inactive region of crossing between the subducted plate and ' $660 \mathrm{~km}$ ' discontinuity, but it is unlikely for the other stations. This interpretation implies, that the discontinuity is depressed only within the subducted plate rather than in a broad zone surrounding it.

Detection of $S_{1070^{\prime}} P$ is the most robust of our results. This signal satisfies all criteria formulated at the beginning of preceding section, and is detected systematically in various data sets. In the synthetics for IASP91, there are no phases that could be mistaken for $S_{1070^{\prime}} P$. The theoretical travel times of $S_{1070^{\prime}} P$ for events 1,3 and $4: 58.8 \mathrm{~s}, 41.3 \mathrm{~s}$, and $49.5 \mathrm{~s}$ match the observed times: $58.8 \mathrm{~s}, 40.4 \mathrm{~s}$, and $50.1 \mathrm{~s}$, respectively. In all cases, when the records of the same events were inspected by NK, the same phase with the same time has been detected at the J-array. Amplitudes of this phase in our data for events in the west $(2,3$, and 4) are larger than the theoretical amplitudes in the synthetics for the $S$ velocity step of $0.2 \mathrm{~km} / \mathrm{s}$. However, our model is without anelastic attenuation, and, if this is taken into account, the observed amplitudes are broadly consistent with the assumed $S$ velocity step. This step is around $50 \%$ of the step at ' $660 \mathrm{~km}$ ' discontinuity and close to that at ' $410 \mathrm{~km}$ ' discontinuity. In the east of the region, $S_{1070^{\prime}} P$ is either relatively weak (event 1), or missing (event 5). This, again, is in agreement with the short-period data obtained by NK. The main problem of this interpretation is caused by the positive values of the differential slowness of $S_{1070^{\prime}} P$ in our data, contrary to the negative values in the synthetics. The discrepancy can be caused by a lateral heterogeneity of the mantle between the discontinuity and the seismograph network, and/or by a topography on the discontinuity. Our data and the data by NK indicate that in a vicinity of event 2 , the discontinuity is uplifted by $60 \mathrm{~km}$, relative to the neighbouring region of events 3 and 4 . The distance between the epicenters of events 2 and 3, 4 is around $200 \mathrm{~km}$. The width of the first Fresnel zone for a period of $10 \mathrm{~s}$ at a depth of $1070 \mathrm{~km}$ is around $200 \mathrm{~km}$, as well. Then, the discontinuity within the region sampled by the records of one event is not a horizontal plane, as assumed in the model calculations, and a strong topography on the discontinuity could contribute to the discrepancy in slowness.

The other detected phases are interpreted as $S_{1170^{\prime}} P$ and 


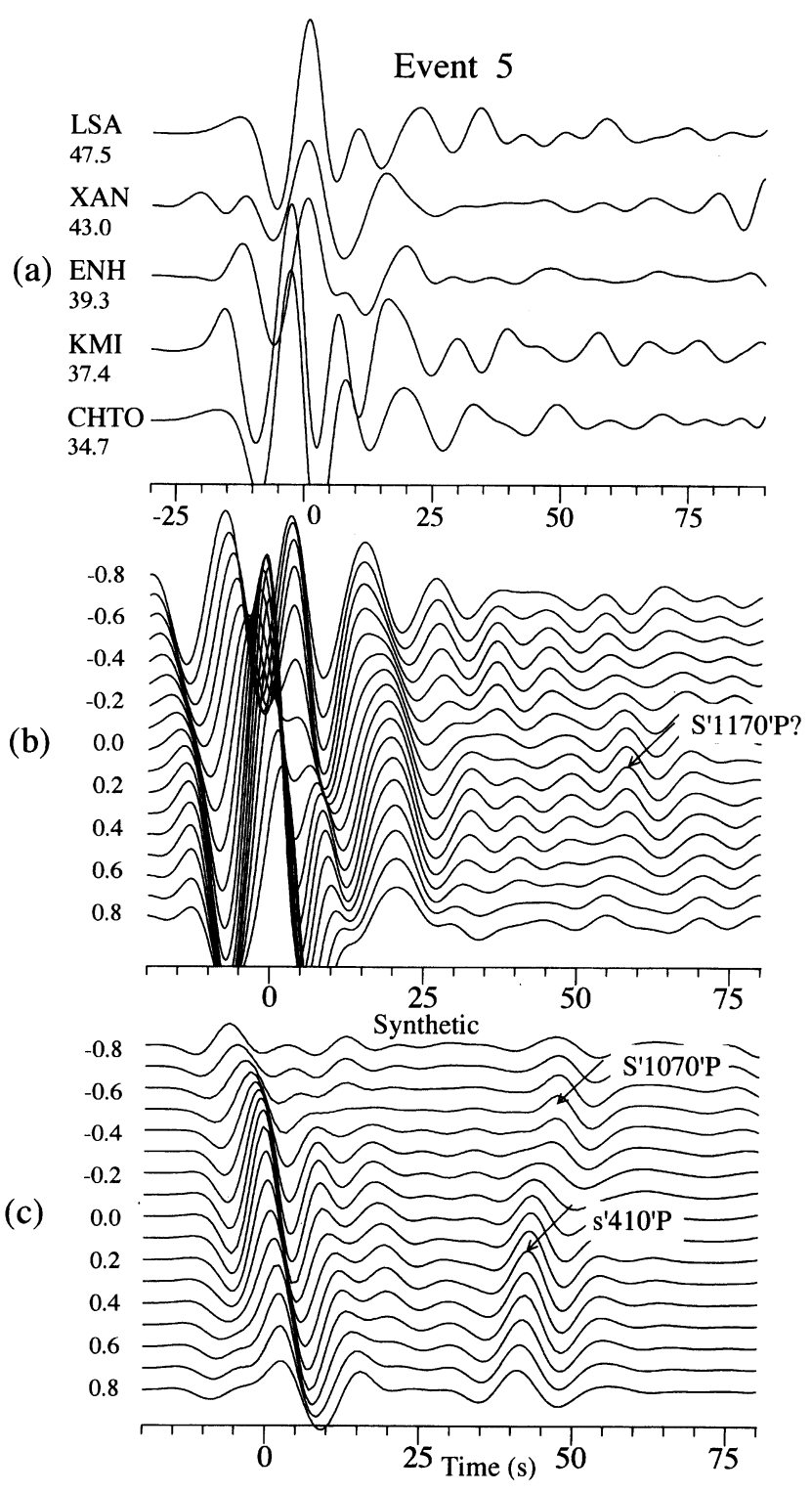

Fig. 11. The same as in Fig. 7, but for the $P$ component traces of event 5 .

$S^{\prime 860^{\prime}} P$. In the records of event $3, s^{\prime} 410^{\prime} P$ could be mistaken for $S_{1170^{\prime}} P$, but this is unlikely for the other events. In the region of event 2 , ' $1170 \mathrm{~km}$ ' discontinuity, like ' $1070 \mathrm{~km}$ ' discontinuity, is uplifted by $60 \mathrm{~km}$. $S^{\prime} 860^{\prime} P$ is relatively clear in the records of event 4 , and a similar phase seems to be present in the records of events 2 and 3 . There are no indications of either ' $860 \mathrm{~km}$ ' or ' $1170 \mathrm{~km}$ ' discontinuities in the short period records of events $2-4$ at the J-array. This can be explained by large widths of the discontinuities. The large width of ' $860 \mathrm{~km}$ ' discontinuity is manifested by the anomalously large dominant period of the assumed $S^{\prime} 860^{\prime} P$. By comparison, ' $1070 \mathrm{~km}$ ' discontinuity is sharp.

It is impossible to identify $S^{\prime} 410^{\prime} P$ in our records, in spite of its strength in the synthetics. There are, at least, two possible reasons for the weakness of $s^{\prime} 410^{\prime} P$ in the actual data. First, contrary to the point source, the waveforms of $S$ radiated upward from the actual source can be different from those radiated downward and adopted for deconvolution. Second, as documented by Vinnik et al. (1996) in easternmost Eura-

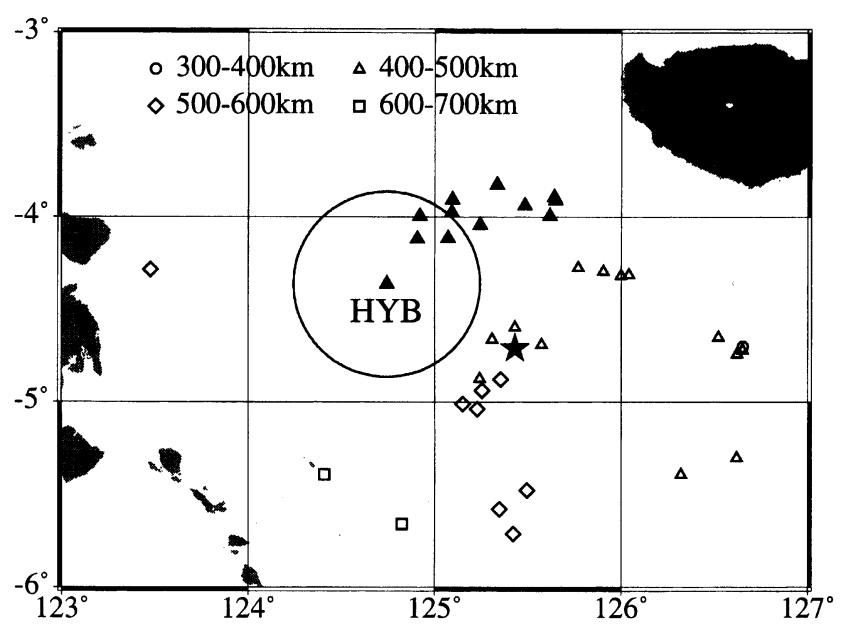

Fig. 12. Region of event 1 (star) with epicenters of deep events and conversion points of $S^{\prime} 660^{\prime} P$ (filled triangles) for all traces shown in Fig. 6, and the first Fresnel zone for HYB. The map suggest, that the first Fresnel zone for HYB may overlap the region of crossing between the subducted plate and ' $660 \mathrm{~km}$ ' discontinuity (currently, without deep seismicity), but this is unlikely for the other stations.

sia, the properties of $410 \mathrm{~km}$ discontinuity in the subduction zones can be unfavourable for generating strong secondary phases. This can be an effect of a strong topography on the discontinuity, or of a broadening of the otherwise sharp discontinuity, due to the presence of water (Wood, 1995).

The results previously obtained for the study region (Niu and Kawakatsu, 1997) and those of the present study are summarized in Fig. 13. Cross-section of the mantle is dominated by the high-velocity body (lower panel, adopted from Sakurai et al. (1995)). It could be suggested that the midmantle discontinuities are not more than the boundaries of the high-velocity or low-velocity anomalies, abundant in the tomographic models of the mantle. As Fig. 13 shows, this explanation is applicable to ' $860 \mathrm{~km}$ ' discontinuity, suggested by our data, but not to ' $1070 \mathrm{~km}$ ' discontinuity, because, as has been shown by NK and is confirmed by the present study, the polarity of ' $1070 \mathrm{~km}$ ' signal corresponds to the $S$ velocity rising rather than decreasing with depth.

Figure 13 demonstrates the only significant discrepancy between the results of $\mathrm{NK}$ and of the present study. The data by NK suggest, that all their observations can be explained by one midmantle discontinuity, which gradually changes its depth from $940 \mathrm{~km}$ in the east to $1080 \mathrm{~km}$ in the west (middle panel). In our data, the phases converted from the different boundaries can be found in the same records of the western events, which means that the three discontinuities are present practically in the same column of the mantle. Moreover, the signals from ' $1070 \mathrm{~km}$ ' and ' $1170 \mathrm{~km}$ ' discontinuities are present in the records of event 1 , in the far east of the region. Thus, the present study favours a few different discontinuities in the midmantle of the target area, rather than one discontinuity with a strongly varying depth. The highvelocity anomaly centered at about $900 \mathrm{~km}$ depth appears to be a flattened continuation of the plate dipping northward along the Sunda arc (Sakurai et al., 1995). Accumulation of the cold and dense material beneath Sunda could be caused 

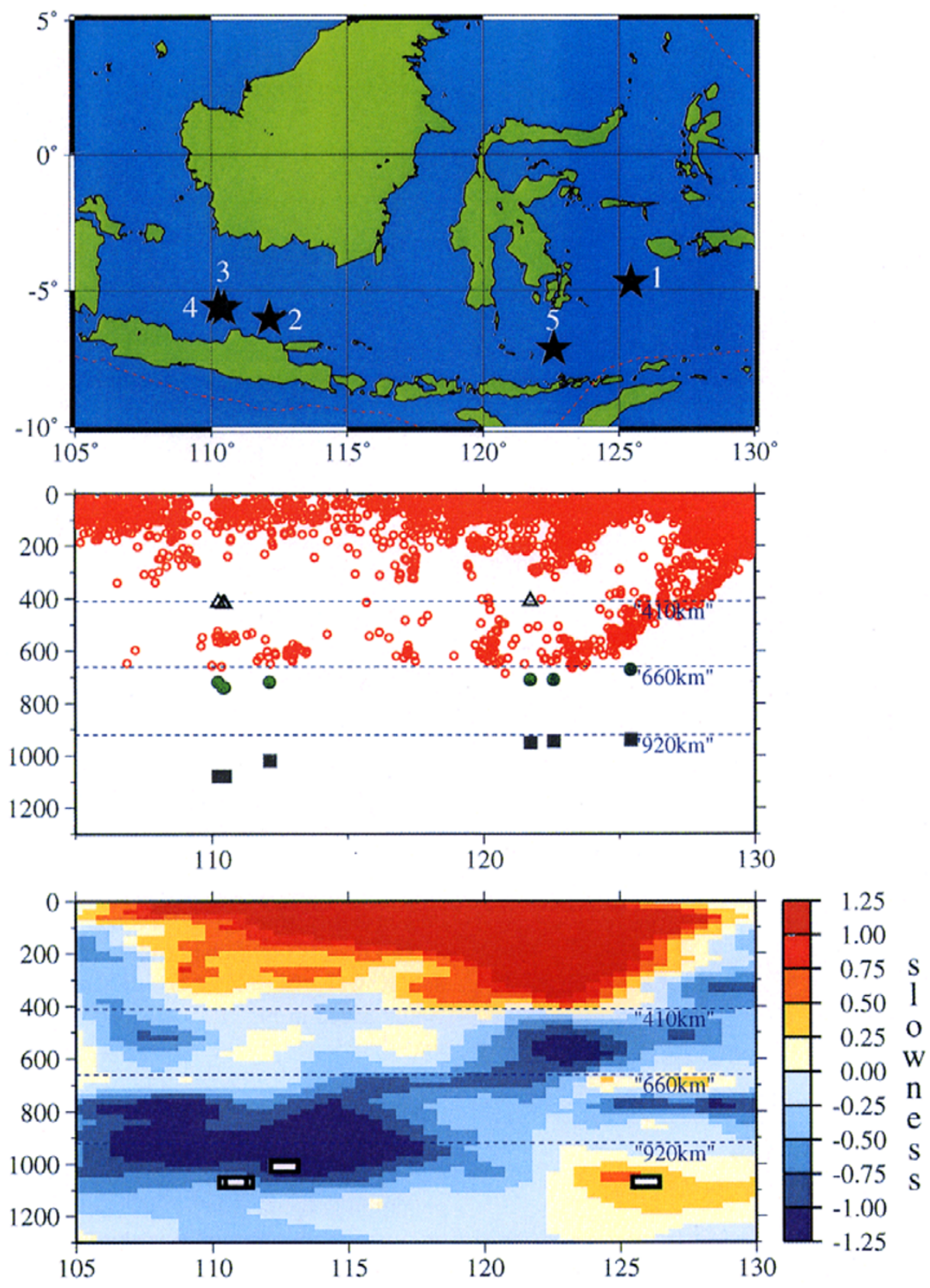

Fig. 13. Summary of seismic data for the mantle beneath Sunda arc: top - map of the region with the epicenters of events in Table 1; middle - vertical E-W cross-section at $-5^{\circ} \mathrm{S}$, with seismicity reported by ISC for 1964-1991, and conversion points (triangles for ' $410 \mathrm{~km}$ ', circles for ' $660 \mathrm{~km}$ ', and squares for ' $920 \mathrm{~km}$ '), inferred from short-period recordings at the J-array, after Niu and Kawakatsu (1997); bottom - the same cross-section with locations of ' 1070 km' discontinuity, according to the present study, and $P$ velocity model by Sakurai et al. (1995).

by a resistance to subduction from ' $1070 \mathrm{~km}$ ' discontinuity. This would imply that ' $1070 \mathrm{~km}$ ' boundary is either a phase boundary with a negative Clapeyron slope or a chemical discontinuity. Other implications of our data, as well as the results for other subduction zones, will be discussed in the papers, now in preparation.

Acknowledgments. This study has been conducted during the visit of one of the authors (LV) to the Earthquake Research Institute, and he thanks the Japanese colleagues for their hospitality. The seismograms are processed with the aid of Seismic Handler, written by K. Stammler. The authors appreciate comments from Ch. Estabrook and an anonymous reviewer.

\section{References}

Aki, K. and P. Richards, Quantitative Seismology: Theory and Methods, 932 pp., W. H. Freeman and Co., 1980.

Barley, B. J., J. A. Hudson, and A. Douglas, S to P scattering at the $650 \mathrm{~km}$ discontinuity, Geophys. J. R. astr. Soc., 49, 773-777, 1982.

Bock, G. and J. Ha, Short-period S-P conversion in the mantle at a depth near 700 km, Geophys. J. R. astr. Soc., 77, 593-615, 1984.

Estabrook, Ch., G. Bock, and R. Kind, Investigation of mantle discontinuities from a single deep earthquake, Geophys. Res. Lett., 21, 1495-1498, 1994

Farra, V., L. P. Vinnik, B. Romanowicz, G. L. Kosarev, and R. Kind, Inversion of teleseismic S particle motion for azimuthal anisotropy in the mantle: A feasibility study, Geophys. J. Int., 106, 421-431, 1991.

Fuchs, K. and G. Mueller, Computation of synthetic seismograms with the reflectivity method and comparison with observations, Geophys. J. R. astr. Soc., 23, 417-433, 1971. 
Goes, S., L. Ruff, and N. Winslow, The complex rupture process of the 1996 deep Flores, Indonesia earthquake (Mw 7.9) from teleseismic P-waves, Geophys. Res. Lett., 24, 1295-1298, 1997.

Kawakatsu, H. and F. Niu, Seismic evidence for a 920-km discontinuity, Nature, 371, 301-305, 1994.

Kennett, B. L. N. and E. R. Engdahl, Travel times for global earthquake location and phase identification, Geophys. J. Int., 105, 429-465, 1991.

Kind, R. and G. Mueller, Computations of SV waves in realistic Earth models, J. Geophys., 41, 142-162, 1975.

Niu, F. and H. Kawakatsu, Direct evidence of the undulation of the $660-\mathrm{km}$ discontinuity beneath Tonga: comparison of Japan and US regional array data, Geophys. Res. Lett., 22, 531-534, 1995.

Niu, F. and H. Kawakatsu, Depth variation of the mid-mantle seismic discontinuity, Geophys. Res. Lett., 24, 429-432, 1997.

Richards, M. A. and C. W. Wicks, Jr., S-P conversion from the transition zone beneath Tonga and the nature of the $670 \mathrm{~km}$ discontinuity, Geophys. J. Int., 101, 1-35, 1990.

Sakurai, T., M. Obayashi, and Y. Fukao, Tomographic image of slab and mantle plume, Program and Abstracts, Seism. Soc. Japan, 1, 624, 1995 (in Japanese)

Vidale, J. E. and H. M. Benz, Upper-mantle seismic discontinuities and the thermal structure of subduction zones, Nature, 356, 678-682, 1992.

Vinnik, L. P., Detection of waves converted from P to SV in the mantle, Phys. Earth Planet. Inter., 15, 39-45, 1977.

Vinnik, L., G. Kosarev, and N. Petersen, Mantle transition zone beneath Eurasia, Geophys. Res. Lett., 23, 1485-1488, 1996.

Wicks, C. W. and M. A. Richards, A detailed map of the 660-kilometer discontinuity beneath the Izu-Bonin subduction zone, Science, 261, 1424$1427,1993$.

Wood, B. J., The effect of $\mathrm{H}_{2} \mathrm{O}$ on the 410-km seismic discontinuity, Science, 268, 74-76, 1995.

L. Vinnik (e-mail: vinnik@synapse.ru), F. Niu (e-mail: niu@eri. u-tokyo.ac.jp), and H. Kawakatsu (e-mail: hitosi@eri.u-tokyo.ac.jp) 